\title{
Global Systematization of Material Objects and the Number of Fundamental Interactions
}

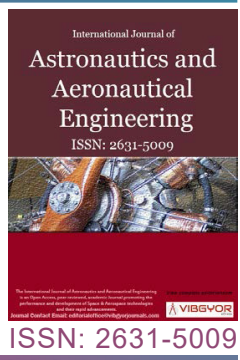

\section{Stabnikov PA* and Babailov SP}

Nikolaev Institute of Inorganic Chemistry, SB RAS, Novosibirsk, Russia

\begin{abstract}
Three approaches explaining the anomalously high rates of star rotation at the peripheries of the galaxies have been formed by present: 1- To introduce the dark matter, 2- To modify Newton's dynamics or to modify the gravitation interaction, 3- To supplement the gravitational potential $\left(F \sim 1 / R^{2}\right)$ with another potential $\left(F \sim 1 / R^{1}\right)$, which decreases no so sharply with an increase in distance and becomes more significant for interstellar distances than classical gravitation. The idea of attractive force inversely proportional to the distance was proposed by JE Tohline (1982) and RH Sanders (1984) to explain the movement of starts in galaxies.

All these approaches explain anomalously high rates of star rotation at the peripheries of galaxies. At present, the majority of astrophysicists maintain the assumption concerning the existence of the dark matter. This approach is especially widespread in popular scientific publications and in television shows. The followers of the second approach are substantially less numerous. The reason is that the mathematical apparatus of different modifications is rather complicated and remains controversial for the majority of scientists. As far as the third approach is concerned, it is supported by only few followers.

To resolve the dispute on which approach of these three is preferable, we decided to involve the most fundamental characteristics of the matter: Its structure and sizes. The sizes of material objects are determined by the four known fundamental interactions (weak, strong, electromagnetic, and gravitational). Every fundamental interaction forms material bodies within definite size ranges. For instance, the strong and weak interactions form elementary particles and atomic nuclei. These interactions weaken at long distances and do not affect the sizes of objects. Electromagnetic interaction forms larger bodies: Atoms, molecules, composite materials, solids. Due to gravitational interaction, dwarf planets, platens, stellar systems and accumulations of stellar systems are formed. In the transition regions, the fundamental interactions competing with each other either are unable to form stable objects (there are no bodies larger than atomic nuclei but smaller than hydrogen atom, which means a size jump of $10^{5}$ ) or promote the formation of a great diversity of objects (in particular, living organisms, asteroids; dwarf galaxies).

If we consider stellar systems, we see that all of them have similar structures with similar dynamics of planet rotation. Considering so large objects as elliptic galaxies, we see that they are also very much alike, but starts at their peripheries rotate with anomalously high rates. However, intermediate formations - dwarf galaxies - are extremely diverse in shapes and structures. This is a valid argument in favor of the third assumption concerning the additional fundamental interaction.
\end{abstract}

\footnotetext{
*Corresponding author: Pavel A Stabnikov, Nikolaev Institute of Inorganic Chemistry, SB RAS, Novosibirsk, Russia

Accepted: May 05, 2021; Published: May 07, 2021

Copyright: (C) 2021 Stabnikov PA, et al. This is an open-access article distributed under the terms of the Creative Commons Attribution License, which permits unrestricted use, distribution, and reproduction in any medium, provided the original author and source are credited.
}

Stabnikov et al. Int J Astronaut Aeronautical Eng 2021, 6:050

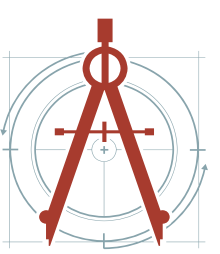


For gravitational interaction, the attractive force is written down as $\mathbf{F}=\left(M_{1} M_{2}\right) \times \gamma / \mathbf{R}^{2}$, while the force for the additional interaction is proposed in the form $F_{\text {add }}=\left(M_{1} M_{2}\right) \times \delta / R$, where interaction constants are $\gamma$ $=6,674184(78) 10^{-11} \mathrm{~m}^{3} \times \mathrm{kg}^{-1} \mathrm{~s}^{-2}$, and $\delta=2.7(0.4) 10^{-31} \mathrm{~m}^{2} \times \mathrm{kg}^{-1} \times \mathrm{s}^{-2}$, while $\mathrm{M}_{1}$ and $\mathrm{M}_{2}$ are the masses of the interacting objects. With these values of the constants, the additional interaction would not have any substantial effect on the movement of planets in stellar systems but it becomes decisive at the periphery of spiral galaxies. This leads to the constant rates of star rotation at the peripheries of galaxies. If we apply thermodynamic virial theory to the interaction between the objects, a stable rotation of objects is possible at any distance from the center for the gravitational potential, while stable formations are impossible for such an exotic potential as $F \sim R^{0}=$ Const. because attraction is absent, and the potential energy of the system is equal to zero. For the proposed potential $F \sim 1 / R$, the formation of stable systems is possible at a limited distance. However, at a distance longer than a definite critical length, stable rotation becomes impossible, which leads to thermodynamically reasonable moving away (recession of galaxies). However, it is not clear yet whether this would be sufficient to explain accelerated expansion of the Universe. At present, this accelerated expansion is considered to be caused by the presence of the exotic dark energy. However, accelerated expansion may also be explained by the effect of some exotic potentials such as $F \sim R^{1}$ or $F \sim R^{2}$.

The goal of the present work is to demonstrate that a theoretical explanation of the motion of matter starting from elementary particles and finishing with galaxies may be provided with the help of four well known potentials and an additional potential with the interaction force $F \sim 1 / R$ without using the idea of dark energy and without various modifications of the laws of physics.

\section{Keywords}

Hierarchical structure of matter from subatomic particle to galaxies, Additional fundamental interaction

\section{Introduction}

The idea of the force of attraction between bodies decreasing inversely proportional to the squared distance was developed by E Halley and I Newton at the end of the XVII century. This allowed explaining how bodies fall on the earth, how the moon and planets rotate in the Solar system. This interaction was called gravitational. Later on, three more additive fundamental interactions were developed for the description of the properties of motion of the matter at shorter distances: The strong, weak, and electromagnetic. The structure and motion of material objects, from atomic nuclei to stellar systems, is well described with the help of all the four interactions. However, for elliptical galaxies, an anomaly was established experimentally: The rates of star rotation with respect to the galactic centre at the periphery of galaxies are almost constant. This makes a substantial difference from the planetary systems, in which the rates of stable motion of planets decrease with an increase in the distance from the central star. It follows from this fact that the gravitational interaction alone is insufficient to explain the anomalous rates of star rotation at the periphery of elliptical galaxies.

This is what promoted the formulation of various approaches, hypotheses and corrections for the explanation of the nature of the anomalous motion of the matter at the super long distanc- es. At first, the existence of the dark matter (DM) was proposed [1,2]: It interacts gravitationally with stars providing their stable rotation around the centre of an elliptical galaxy with the constant rate and allows explaining high relative velocities of galaxies in their accumulations. Later various modifications of Newton's laws were developed [3-9], even the modification of the gravitational potential itself was proposed, with the help of a correction adopted from the Yukawa potential $[10,11]$. The author of [12] proposed to explain all this through the influence of the increased density of the cosmic radiation at the periphery of galaxies, which enhances the interaction of gravitons, according to the assumption of the author. The approach is also known that involves the introduction of the fifth additional discrete fundamental interaction which becomes significant at super long distances [1318]. The attraction force for this interaction should be $F \sim 1 / R$, because only in this case the stars will steadily rotate with the constant rate at the peripheral regions of galaxies. A more detailed consideration of the dependence of the rates of stable rotation of satellites on the kind of potential $\phi \sim R^{n}$ is given in $[17,18]$. Many other unusual hypotheses explaining anomalous rates of star rotation at the periphery of galaxies were proposed (for example, see $[12,19-23])$.

According to the authors of all these various 


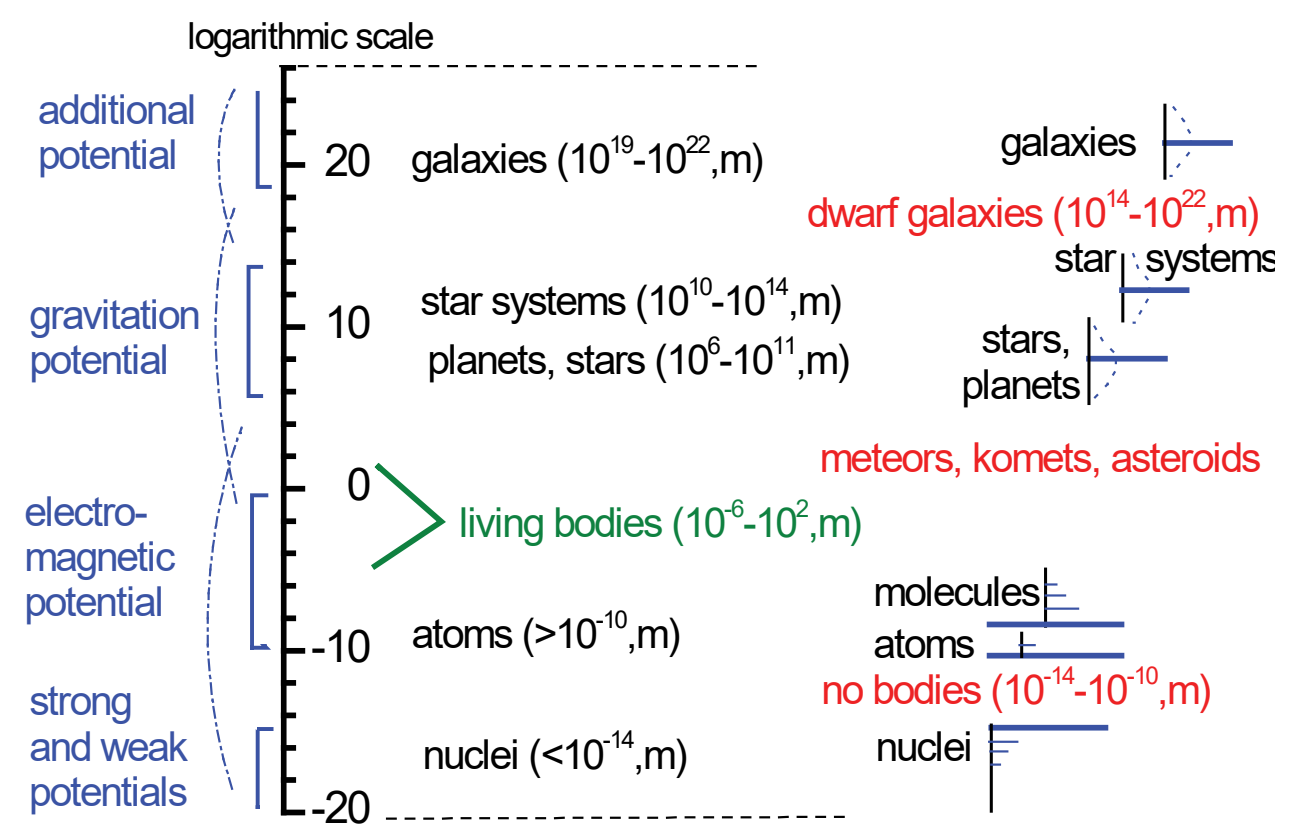

Figure 1: Generalized notion of the effect of fundamental interactions on the formation of stable material objects. $\checkmark$ the range of the influence of a potential.

assumptions, a hypothesis proposed by an author is the only correct one, providing an excellent description of the features of star rotation at the peripheral regions of elliptical galaxies. Naturally, those authors subject all other assumptions to criticism. A discussion of some approaches is given in Supplementary materials. However, there is still a controversy about which of the approaches supplementing the gravitational interaction at super long distances is the best and the most correct one. The present work deals with this problem. The basis is the most fundamental property of the matter: Its structure.

\section{Description of Matter Structure from Minuscule to Gigantic Sizes}

A scheme of the hierarchical system was proposed in $[23,24]$ to describe the global structure of the matter. The scheme of the structure of matter that was developed on the basis of those works is shown in Figure 1 . The objects within the size range from $10^{-20}$ to $10^{26} \mathrm{~m}$ (the sizes of all bodies known at present) are represented in the logarithmic scale. The significance bands and the ranges of influence of the fundamental interactions including the additional potential are shown at the left-hand side in the blue colour. The names of objects with the broad variety of shapes and sizes including dwarf galaxies are shown at the right-hand side in the red colour. The discrete nature of nuclei, atoms and molecules is also shown at the right-hand side in the blue colour. Planets, stars, planetary systems and galaxies different in sizes but close in shapes are shown on top.

The smallest bodies in Figure 1 are elementary particles and atomic nuclei. The main feature of these formations is indistinguishability of identical objects. To describe their properties and stability, two fundamental interactions were developed: Strong and weak. These interactions are prevailing at distances up to $10^{-14} \mathrm{~m}$. Electromagnetic interaction is also used to describe the properties of nuclei; In particular, this interaction allows explaining the radioactive decay of heavy nuclei. However, in the majority of cases this interaction is less significant within this distance range than the strong interaction. The next larger material formations are atoms. Their sizes are much larger, not less than $10^{-10} \mathrm{~m}$. But there are no material stable formations with the size from $10^{-14}$ to $10^{-10} \mathrm{~m}$ (six orders of magnitude). This is so-called transition interval which may be explained as follows: The strong interaction is no more able to generate objects on this scale, while the electromagnetic interaction is yet unable to generate stable formations.

The electromagnetic interaction forms material objects with a size from hydrogen atom $\sim 10^{-10} \mathrm{~m}$ to the sizes of solid bodies. The same atoms are completely identical. Larger formations-molecules 
DISTRIBUTION OF DARK MATTER IN NGC 3198

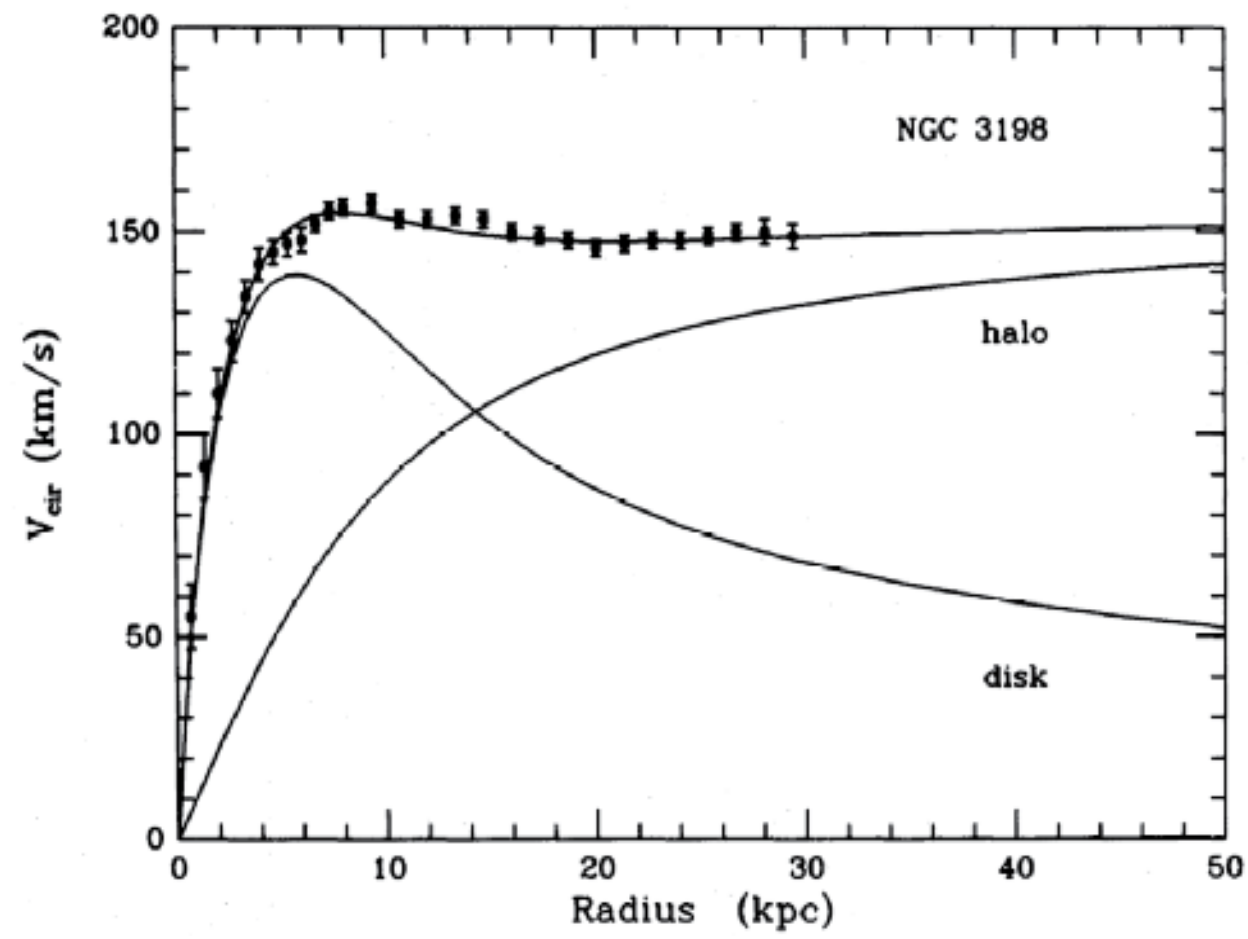

Figure 2: From site http://present5.com/presentation/-30898083_48433107/image-13.jpg. Copies of a similar drawing can be found in the works $[11,17]$.

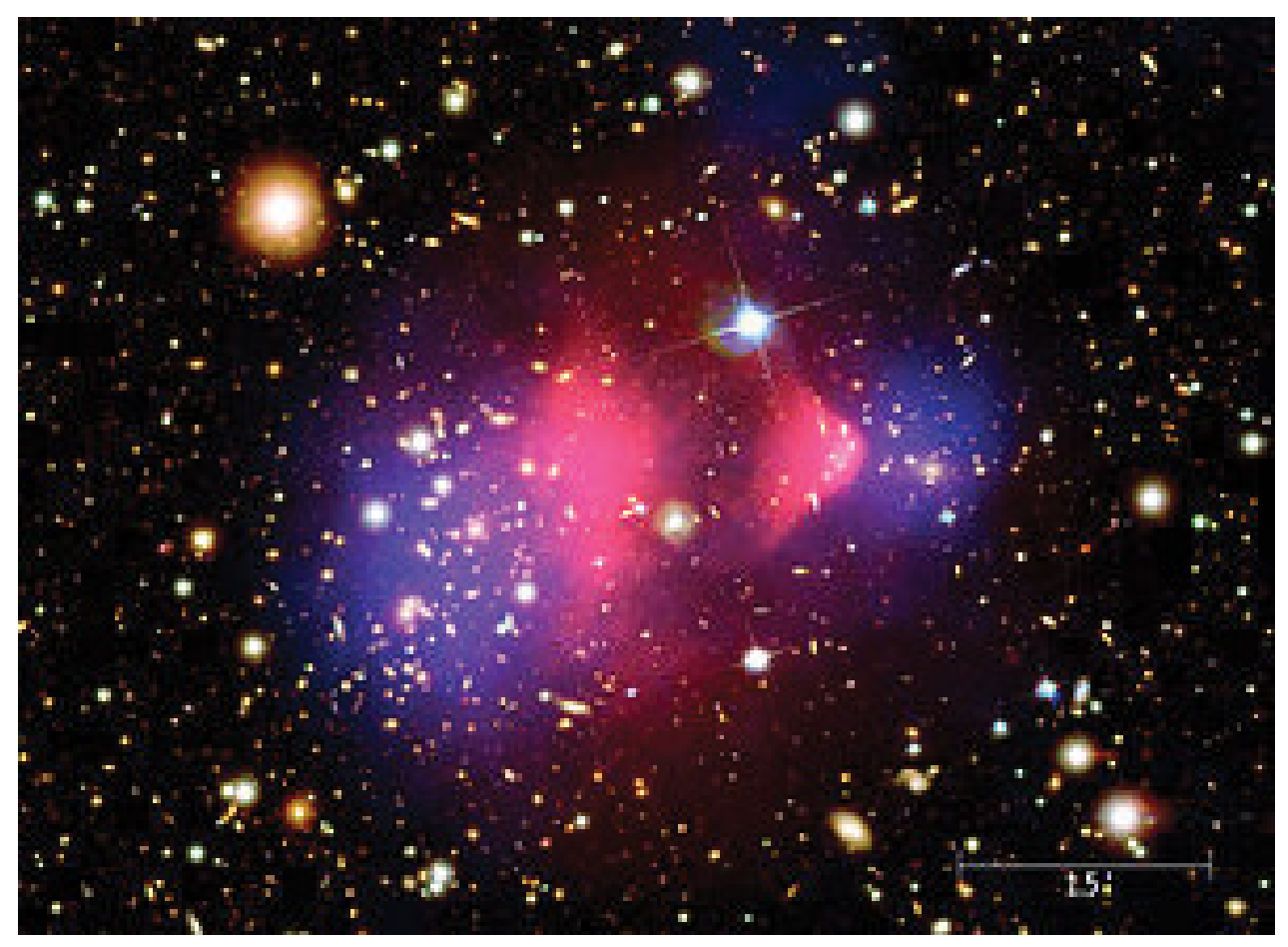

Figure 3: The Bullet Cluster. X-ray image (pink) over a visible-light image of galaxies, and DM distribution calculated from gravitational lensing (blue). A color photograph can be found at: https://en.wikipedia.org/wiki/ Bullet_Cluster.

having the same composition and structure are indistinguishable too. As the sizes of the objects in- crease (polymers, nanoparticles, composite materials, crystals, solids), their composition and struc- 
ture become more and more diverse. Usual crystal sizes are from $10^{-2}$ to $\sim 1 \mathrm{~m}$. This fundamental interaction weakens for larger objects because of the compensation of the charges of different signs and spontaneous charge leakage. In other words, the size range from $10^{-10}$ to $1 \mathrm{~m}$ (about ten orders of magnitude) is assigned for the electromagnetic interaction in our world. This interaction is also pronounced at larger distances (lightnings, magnetic fields of planets and stars etc.) but for the equilibrium systems it becomes weaker that the gravitational interaction.

The interval from $10^{-6} \mathrm{~m}$ ((living cell size) to $10^{2}$ $\mathrm{m}$ (the height of the largest tree-sequoia) should be stressed specially. This is so-called life interval. Within this interval, the significance of the electromagnetic action decreases and the role of gravitation increases. At the very beginning of this interval, quantization of the objects is typical, which allows living organisms to encode the genetic information at the molecular level. The origination and existence of life are possible in this range, in which one fundamental interaction comes to replace the other and in which the diversity of material objects is possible. The occurrence of the life interval in our Universe is a consequence of the discreteness of the electromagnetic and gravitational fundamental interactions and the partial overlap of the regions where their effect on the motion and formation of material objects is noticeable.

The gravitational interaction is noticeable for the bodies that possess the mass is their sizes are larger than $10^{-2} \mathrm{~m}$ (experiments of $\mathrm{H}$ Cavendish). With an increase in the mass of bodies, the gravitational interaction increases. We may accept that the prevalence of the gravitational interaction starts from the size for which it is able to form a spherical shape of cosmic bodies. One of these not very large spherical solid formations is Ceres, a body within the asteroid belt, with a radius of $4.9 \times 10^{5} \mathrm{~m}$. With this approach, the interval of the existence of material objects from 1 to $10^{6} \mathrm{~m}$ (six orders of magnitude) is a transition interval in which the significance of one of these fundamental interactions is replaced by the other. According to the modern notions, the gravitational interaction is determinative till the boundaries of the observable Universe, about $1.3 \times 10^{26} \mathrm{~m}$, that is, twenty orders of magnitude as a total. This is two times more than the range assigned for the significance of the electromagnetic interaction.
If 20 orders of magnitude of object sizes are assigned in our world for the prevalence of the gravitational interaction, this interaction should generate similar material formations within this range. However, it is well known that there are two types of stable material formations in our Universe: planetary systems, with the sizes from $10^{10}$ to $10^{14} \mathrm{~m}$, and elliptic galaxies, with the sizes from $10^{19}$ to $10^{22}$ $\mathrm{m}$ (Figure 1). The structures and dynamics of the motion of inner parts are different in these objects. In addition, within the size range from $10^{14}$ to $10^{19}$ $\mathrm{m}$ (five orders of magnitude) there are dwarf galaxies of different shapes: Elliptic, spiral, spheroidal, irregular tufted. The number of dwarf galaxies is much larger than the number of elliptic ones. Only in the vicinity of our Milky Way, 14 dwarf galaxies have already been discovered. The cross size of the largest of them, the Big Magellanic cloud, is about 7000 light years $\left(6.62 \times 10^{19} \mathrm{~m}\right)$, which accounts for about $7 \%$ of the diameter of the Milky Way galaxy $\left(9.46 \times 10^{20} \mathrm{~m}\right)$. Dwarf galaxies with the size about 150 light years and even smaller and dim dwarf galaxies called hobbit galaxies were also discovered. A potential dwarf galaxy may be composed of only several star systems. It should be noted that the boundary of heliosphere of our Solar System where the solar wind slows down is only $75-90$ astronomical units $\left(1.2-1.4 \times 10^{-3}\right.$ light years, or 1.1-1.3 $\times 10^{13}$ $\mathrm{m})$ according to the data obtained from the Voyager-1.

The hierarchical nature of material formations resembles the structure of nesting dolls. The major principle of their structure is in the little being a part of a larger, and this larger being included into even larger object. The discreteness is clearly pronounced in the structure of nesting dolls: The transition from the size of one doll to the size of another is jump wise. However, the nature of the structure of matter is more complicated. A new level unites not a single object as in nesting dolls but a great number of objects. For instance, for the transition from nuclei to atoms, the new formation is composed of a nucleus and several electrons. The volume of the newly formed object is much larger than the sum of its components. Because of this, the density of any new, larger material formation is usually lower than the density of each of its components. In other words, a larger object is formed from the empty space and a definite number of denser components. Discrete fundamental interactions were proposed by the preceding generations 
of physicists to describe this structure of the matter.

\section{Numerical Value of $\delta$ and the Possibilities of this Kind of Interaction}

For the additional interaction, the force is proportional to $F \sim 1 / R$ [13-18]. Only this kind of interaction provides constant rates of satellite rotation with an increase in the distance from the centre. The value of $\delta \sim 1.7 \cdot 10^{-31} \mathrm{~m}^{2} \times \mathrm{kg}^{-1} \times \mathrm{s}^{-2}$ was proposed for this kind of interaction in [15] on the basis of logical considerations. The value of $\delta \sim 2.7 \cdot 10^{-31} \mathrm{~m}^{2}$ $\times \mathrm{kg}^{-1} \times \mathrm{s}^{-2}$ was proposed in [17] on the basis of the plot of star rotation around the galaxy centre. The reliable value $\delta=2.7(4) \cdot 10^{-31} \mathrm{~m}^{2} \times \mathrm{kg}^{-1} \times \mathrm{s}^{-2}$ was obtained in [16] by fitting the rotation of stars in 60 galaxies. For a larger scale involving many galaxies, no stable formations are observed (or have been discovered by present). Because of this, there are not reasons to introduce any additional fundamental interactions.

It should be noted that the force of the gravitational attraction of the Earth to the Sun is equal to $3.54 \times 10^{22} \mathrm{~N}$, while the force of attraction calculated for the additional potential is $2.1 \times 10^{13} \mathrm{~N}$, which is 9 orders of magnitude weaker. This would not have any effect on the orbit of the Earth. The boundary of the heliosphere of the Solar System extends to $\sim 1.3 \times 10^{13} \mathrm{~m}$. At this distance, the gravitational interaction is still stronger than the additional one. Only at a distance of $8 \mathrm{kpc} .\left(2.47 \times 10^{20}\right.$ $\mathrm{m})$ from the Sun, the force of the gravitational interaction will become equal to the force of the additional interaction, which will be equal to $1.3 \times 10^{4}$ $\mathrm{N}=1.33 \times 10^{3} \mathrm{~kg}(\mathrm{f})$ for such formation as the Earth. It follows from these considerations that the additional interaction with $\delta=2.7(4) \cdot 10^{-31} \mathrm{~m}^{2} \times \mathrm{kg}^{-1} \times \mathrm{s}^{-2}$ will have almost no effect on the structure of stellar systems. In the central part of an elliptical galaxy, gravitational interaction is prevailing, while closer to the periphery (at a distance of more than $8 \mathrm{kpc}$ ) the additional interaction becomes prevailing.

According to the laws of thermodynamics, a system will be stable only if the total energy of interaction is below zero: $U=E_{k i n}+E_{p o t}\left(E_{k i n}>0, E_{p o t}<0\right)$ [25]. Since the rates of satellite rotation at the periphery of galaxies are almost constant, then, $E_{k i n}=m^{2} / 2$ is the same for any satellite with the same mass. The potential energy of satellite attraction for the additional interaction $(F \sim 1 / R)$ is expressed as $E_{p o t}=\delta \times$
$M_{1} M_{2} \times \operatorname{Ln}(R)[17,18]$. In other words, the potential energy of a satellite varies from a negative value to a positive one similarly to the function $\operatorname{Ln}(R)$. (This is a substantial difference from the gravitational interaction, for which $E_{\text {pot }}=-\gamma \times M_{1} M_{2} / R$ permanently decreases in the absolute value with an increase in the distance but remains a negative value). The system will become unstable, and the stellar system will be unable to stay at the edge of a galaxy after $E_{\text {pot }}$ becomes less than $E_{k i n}$ in the absolute value. Because of this, the laws of thermodynamics define the sizes of galaxies. According to the virial theorem, for the interaction with the force $F \sim 1 / R, E_{\text {kin }}$ $=M_{2} V^{2} / 2=\delta \times M_{1} M_{2} / 2$, and $E_{p o t}=\delta \times M_{1} M_{2} \times \operatorname{Ln}(R)$ $[17,18]$. It follows from these relations that a galaxy will be thermodynamically stable if $R<0.607$. This value may be chosen as the Galactic Universal Unit (GUU). The diameter of our Milky Way galaxy is $2.9 \times 10^{4} \mathrm{kpc}$, and the diameter of the largest known galaxy C 1101 is $1.8 \times 10^{5} \mathrm{kpc}$. Because of this, GUU value may be estimated as slightly larger than the radius of $C 1101$, that is, $\sim 5 \times 10^{5} \mathrm{kpc}$. The logarithmic dependence of the potential energy of additional interaction on the distance explains the finite sizes of galaxies. This estimation was carried out without taking into account the gravitational interaction, which is small for super long distances, and assuming that the laws of thermodynamics are correct at super long distances.

It should also be noted that for additional interaction, the farther away from the outer border of the galaxy the body is, the more profitable it is to move away. This potentially explains the expansion of the Universe. The additional interaction is introduced for super long distances, so this approach does not reject the possibility of the existence of relatively not very large objects such as black holes, neutron stars, quasars etc. Our Universe is also rich in intergalactic gas and dust. These objects were not included into consideration because of their low density and uncertainty in shapes and sizes.

\section{Conclusion}

The nature of the general structure of the matter presented in Figure 1, with the alternation of the intervals with similar structures of the objects and the intervals with the great diversity of the shapes and sizes of the objects, suggests that the four fundamental interactions are insufficient for the description of the properties and structure of the matter within the whole range of object sizes 
from $10^{-20}$ to $10^{26} \mathrm{~m}$. This conclusion was made on the basis of traditional scientific logic relying on the most fundamental property of the matter: Its structure.

All known approaches developed for the explanation of the constancy of the rates of star rotation at the periphery of galaxies are unable to explain the great diversity of the shapes of dwarf galaxies. Only with the help of the additional interaction, this feature of the structure of the matter may be easily explained by the competition between two potentials with different manners of a decrease in the interaction force depending on the distance.

So, with the Help of the Additional Interaction with the Constant $\Delta$, it is Possible:

1. To explain the great diversity of dwarf galaxies.

2. To explain the constancy of the rates of star rotation at the periphery of galaxies.

3. To continue the traditional approach developed by many generations of physicists for the description of the properties of the matter with the help of fundamental interactions.

4. To recover the previous notions of our Universe without the dark matter.

5. To explain the accelerated expansion of the Universe, substantially smaller total amount of the dark energy (DE) is necessary than the value accepted at present.

Thus, it is possible to explain all the properties of material objects from elementary particles to the boundaries of the observable Universe with the help of four well known interactions plus one additional interaction, and with the help of the idea of the influence of the DE on the expansion.

\section{References}

1. Zwicky F (1937) On the masses of nebulae and clusters of nebulae. Astroph J 86: 217.

2. Rubin V, Ford W (1970) Rotation of the andromeda nebula from a spectroscopic survey of emission regions. Astroph J 159: 379.

3. Finzi A (1963) On the validity of newton's law at a long distance. Monthly Notices Roy Astronom Soc 127: 21-23.

4. Milgrom M (1983) A modification of the newtonian dynamics as a possible alternative to the hidden mass hypothesis. Astrophys J 270: 365-370.

5. Bekenstein JD (2004) Relativistic gravitation theo- ry for the modified newtonian dynamics paradigm. Phys Rev D 70: 083509.

6. Brownstein JR, Moffat JW (2006) Galaxy rotation curves without non-baryonic dark matter. Astroph J 636: 721-741.

7. Hossenfelder S (2008) Bimetric theory with exchange symmetry. Phys Rev D 78: 044015.

8. Wei L, Lixin X (2013) Viscous generalized chaplygin gas as a unified dark fluid. Eur Phys J 73: 2471.

9. Portnov Yu A (2014) Obtaining galaxy rotation curves without dark matter. Grav Cosmol 20: 279-281.

10.Brandao CSS, de Araujo JC (2010) Probing brownstein - moffat gravity numerical simulations. Gen Relativ Gravit 42: 623-632.

11.Brandao CSS, de Araujo JC (2008) Galactic disks in theories with yukawian gravitational potential. Project Cjsmology.

12.Clark MJ (2018) Cosmic rays and galactic rotation curves. Astrophys Space Sci 363: 1-12.

13.Tohline JE (1982) Stabilizing a cold disk with a $1 / r$ force law. Proceedings of the Symposium, Besancon, France (A83-49201 24-89).

14.Sanders RH (1984) Anti-gravity and galaxy rotation curves. Astronomy and Astrophysics 136: L21-L23.

15. Rimsky-Korsakov AA (2003) Variant of modification of gravitational forces. Proceedings of the Radium Institute Khlopin St Petersburg 10: 65-69.

16. Puga VA (2014) Multidimensional gravitational interaction. Galaxy rotation curves. JETP 146: 500-512.

17.Stabnikov PA, Babailov SP (2017) An addition to the classic gravity interstellar interactions. J Astrophys Aerospace Technol 5: 1-4.

18.Stabnikov PA (2018) The framework in which matter develops. Palmarium Academic Publishing, 81.

19. Ryabov VA, Tsarev VA, Tskhovrebov AM (2008) Search for dark matter particles. Yspekhi Phys Nauk 51: 1091-1121.

20.Zasov AV, Saburova AS, Khoperskov AV, Khoperskov SA (2017) Dark matter in galaxies. Physics-Uspekhi 60: 3-39.

21.Gianfranco B, Dan H (2016) History of dark matter. Rev Mod Phys, 2.

22.Kroupa P (2012) The dark matter Crisis: Falsification of the current standard model of cosmology. Astronomical Society of Australia 29: 395-433.

23.Ivanov BN (1986) Laws of physics. Vyssh Shk, Moscow, 336.

24. Mazalov LN (2010) Physical foundations of measurements. Novosibirsk, Russia, 2.

25.Landau LD, Lifshitz EM (2001) Mechanics, Pergamon Press, Moscow, 1. 


\section{Supplementary Materials}

\section{Problems of other approaches}

The introduction of the dark matter (DM). In 1932, astronomer F Zwicky investigating the accumulations of galaxies in the Berenice's Hair constellation estimated the mass of galaxies, their relative velocities of motion, attraction energy $\left(\mathrm{E}_{\mathrm{pot}}\right)$ and kinetic energy $\left(T_{\text {kin }}\right)$. However, the results were in clear contradiction with the laws of thermodynamics. For instance, according to the virial theorem for a stable orbital system, for the gravitational interaction $\left(F \sim R^{-2}\right)$ the relation between $T_{\text {kin }}$ and $E_{\text {pot }}$ is written as $2 \mathrm{~T}_{\text {kin }}=-\mathrm{E}_{\text {pot }}$. $[17,18,25]$. Then, the total energy of such a system $U=E_{\text {pot. }}+T_{\text {kin. }}=1 / 2 E_{\text {pot. }}$ is a negative value, which points to the thermodynamic stability of the system. Moreover, this system has a definite factor of safety. If we add a definite amount of the kinetic energy, less than $1 / 2 \mathrm{E}_{\text {pot }}$ in the absolute value, to a satellite, the system would not be destroyed, only the satellite will change its orbit to the elliptical one. If the additional kinetic energy is larger than $1 / 2 \mathrm{E}_{\text {pot }}$ in the absolute value, the total energy $U$ will become positive. In this case, the system will be destroyed: the satellite will fly away.

However, F Zwicky established that the velocities of galaxies in the Berenice's Hair constellation exceed the estimated values by a factor of 50-160 [1]. This means that the motion of the galaxies with respect to each other does not fit a stable model. The galaxies fly away from each other. These were the first evidences of the expansion of the Universe. But F Zwicky was a follower of the stable model. Because of this, to rescue the stability of the Universe, he put forward rather simple assumption concerning the existence of the DM. Indeed, some DM will cause an increase in the potential energy of attraction $\mathrm{E}_{\text {pot }^{\prime}}$ which will allow us to make the total energy negative, typical for stable models. However, this will be true only in the case if the DM would not make any contribution into the kinetic energy. In other words, the DM should interact gravitationally with usual matter causing an increase in the stability of a cluster of galaxies but it should be immovable. At that time, more than 85 years ago, there was a hope that the DM would be detected experimentally in the future, would be possible to explain its zero contribution into the kinetic energy.

It should be noted that a comparison between the potential and kinetic energies of any body is equivalent to a comparison between the gravitational and inertial masses of that body (or accumulation of bodies). However, the identity of the gravitational and inertial masses of usual bodies had been demonstrated experimentally in 1867 by I. Newton with the accuracy of $10^{-3}$. Later, in 1901, E Lorand had enhanced the accuracy of coincidence to $10^{-8}$. A. Einstein put the identity of the gravitational and inertial masses into the basis of the general relativity, which is now the major theory of gravitation, well confirmed by observations. The DM, which presumably possesses only the gravitational interaction, differs from the usual matter substantially and cannot be described within the framework of the general relativity.

Potentially, F Zwicky possessed two more opportunities to explain anomalously large relative velocities of the motion of galaxies: Updating of the gravitational potential so that the force of this interaction will decrease slower than $\sim 1 / R^{-2}$ at super long distances, or the introduction of an additional potential of attraction, with the force proportional to $1 / R^{-1}$. The corrections of this kind would allow us to conserve the stability of the accumulations of galaxies, in spite of their rather high velocities, but these versions of updating were much more complicated than just the introduction of unusual additional kind of matter, which has not been detected yet.

Somewhat later, after the invention of spectral telescopes, the rates of rotation of the stars in galaxies were established with the help of C. Doppler's effect. The results are shown in Figure 2.

This is a generalized drawing characteristic of the majority of spiral galaxies. In the vicinity of the centre, up to a definite critical region $\mathrm{R}_{\mathrm{b}} \approx 8 \mathrm{kps}$, an increase in the rates of star rotation around the centre is observed. This region of the central part of the galaxies is called Bulge. At a distance longer than $R_{b}$, the density of the stellar matter in the galaxy decreases, which is confirmed by astronomical observations. But in this case the rates of star rotation should decrease according to J Kepler's laws, similar to the rates of planet rotation in the Solar System (disk curve, Figure 2). This would be in complete agreement with the prediction of the virial theory. However, at the distances longer than $R_{b}$ the rates of star rotation remain almost constant up to the galactic edge. It follows from this that the larger is the distance of a star from $\mathrm{R}_{\mathrm{b}}$, the more its 
motion deviates from J Kepler's laws and from the predictions of the virial theory. In other words, it followed from experiments that the galaxies should be unstable. To rescue the stability of galaxies, $\mathrm{V}$ Rubin, W Ford assumed in [2] that the DM proposed by $\mathrm{F}$ Zwicky exists also inside the galaxies.

However, for the rates of star rotation around the galaxy centre at distances longer than $R_{b}$ to be nearly constant, it is necessary that the density of the DM increases from the centre to the periphery (halo curve, Figure 2). One more unusual property of the DM follows from this: the visible matter should attract to the DM but the DM itself should be repulsed from the visible matter because the density of the DM is minimal in the centre of a galaxy (Figure 2). This kind of the distribution of the DM in a galaxy resembles a divergent lens, so the DM should not focus the light passing by but scatters it instead. The distribution of the baryon matter in the galaxy resembles a focusing lens, so light lensing by galaxies may be explained only by the presence of usual matter.

In general, the DM was introduced to conserve the stability of the Universe and to explain anomalously high rates of star rotation at the edges of the galaxies. This purely logical introduction gave DM unusual properties: It had to be immobile (to have no kinetic energy $T_{\text {kin. }}$ ) and to possess unusual attraction-repulsion properties.

At present, the most valid proof of the existence of the DM in reality is considered to be the interpretation of the photographs of a collision between two galaxies in the Bullet Cluster (Figure 3).

Two colliding galaxies are shown in Figure 3. Only the usual matter (stars, overheated gas) is visible. The DM is not visible, it was drawn additionally. However, it is unknown who was that astrophysicist of genius (or a team of astrophysicists) who did this. An explanation is given: The DM was drawn on the basis of the lensing effect. This procedure is not described. In any case, the drawing is interpreted as follows. Before the collision, both the visible matter and the DM in each galaxy had the same velocities of motion. During the collision, the visible matter of both galaxies hampered the counter motion of each other, while the DM of both galaxies passed through the counter galaxy without hindrance. Because of this, the visible matter lagged behind the motion of the DM. This follows from the images edited by additional drawings. However, this approach renders both gravitational and inertial masses to the DM. This contradicts the assumption made by $\mathrm{F}$ Zwicky that the DM possesses only the gravitational interaction and makes no contribution into the kinetic energy (does not possess inertial mass). Here is a contradictory interpretation of DM characteristics. So, it should be stressed that the notion of the DM is very diverse, flexible and inconsistent. Of course, the notion of the DM may be used, but then one should understand that sometimes it would be necessary to exceed the bounds of traditional scientific logic.

The artificial introduction of the DM possessing unusual properties was not approved by some astrophysicists. Theoretical reasonableness of the introduction of DM in our Universe is under extensive discussions at present [19-22]. In addition, unquestionable data confirming the actual existence of the DM are absent at present. Because of this, alternative approaches are proposed to explain the anomalous motion of stars in the peripheral regions of galaxies [3-12]. By present, more than 30 alternative models correcting I. Newton's laws and gravitation at long distances have been developed. However, it should be noted that these approaches in their essence are linear corrections to the laws of classical mechanics. This is why these approaches are unable to explain a jumping transition from stellar systems to elliptic galaxies and a great diversity of the shapes of dwarf galaxies. 